\title{
STABILITY OF NEURAL NETWORKS
}

\author{
Grienggrai Rajchakit
}

\begin{abstract}
We develop a new approach to the stability analysis of Hopfield-type neural networks with time varying delays in the presence of impulses. With the new approach, we improve and generalize some previous works of other researchers. We study stability of equilibrium points of impulsive systems which are either a generalization of those existing or new. This paper deals with the problem of delaydependent stability criterion of delay-difference system with multiple delays of Hopfield neural networks. Based on quadratic Lyapunov functional approach and free-weighting matrix approach, some linear matrix inequality criteria are found to guarantee delay-dependent asymptotical stability of these systems.
\end{abstract}

Keywords - Hopfield neural networks; Time-varying Delay; Stability; Quadratic Lyapunov functional approach.

\section{INTRODUCTION}

Hopfield neural networks have been extensively studied in past years and found many applications in different areas such as pattern recognition, associative memory and combinatorial optimization. Such applications heavily depend on the dynamical behaviors. Thus, the analysis of the dynamical behaviors is necessary step for practical design of neural networks. A neural network is a network that performs computational tasks such as associative memory, pattern recognition, optimization, model identification, signal processing, etc. on a given pattern via interaction between a numbers of interconnected units characterized by simple functions. From the mathematical point of view, an artificial neural network corresponds to a nonlinear transformation of some inputs into certain outputs. Many types of neural networks have been proposed and studied in the literature and the Hopfield-type network has become an important one due to its potential for applications in various fields of daily life. The model proposed by Hopfield, also known as Hopfield's graded response neural network is based on analog circuit consisting of capacitors, resistors and amplifiers. Among the most popular models in the literature of artificial neural networks (see, e.g., [1-7]) is the continuous time model described by a system of ordinary differential equations. The multiple models are still multiple linear models with different parameters, and neural network is used only to compensate for the modeling error of linear model. In this case, the nonlinear system should

Grienggrai Rajchakit is with the Department of Mathematics, Faculty of Science, Maejo University, Chiang Mai 50290, Thailand not be very complex, and too big modeling error between the system and linear model is forbidden. Dynamical systems are often broadly classified into two categories: continuous time systems or discrete time systems. Recently there has been introduced a somewhat new category of dynamical systems which is neither purely continuous time nor purely discrete time ones; these are called dynamical systems with impulses (see for instance [8-11] and references therein). Stability conditions for various types of stability of neural networks problems such as complete stability, asymptotic stability, absolute stability and exponential stability have been studied extensively. One should underline the fact that stability properties of a neural network basically depend on the intended problems. For example in the solution of optimization problems, the neural network must be designed to have only one equilibrium point and this equilibrium point is globally stable. See more details in [12-15] and references given therein.

In this paper, we consider delay-difference system with multiple delays of Hopfield neural networks of the form

$$
u(k+1)=-C_{i} u(k)+\sum_{i=1}^{m} B_{i} S\left(u\left(k-h_{i}\right)\right)+f,
$$

where $u(k) \in \Omega \subseteq \mathbf{R}^{n}$ is the neuron state vector, $0 \leq h_{1} \leq \ldots \leq h_{m}, \quad C_{i}=\operatorname{diag}\left\{a_{1 i}, \ldots, a_{n i}\right\}, \quad c_{i} \geq 0$, $i=1,2, \ldots, n$ is the constant relaxation matrix, $B_{i}, i=1,2, \ldots, m$ are $n \times n$ constant weight matrices, $f=\left(f_{1}, \ldots, f_{n}\right) \in \mathbf{R}^{n}$ is the constant external input vector and $S(z)=\left[s_{1}\left(z_{1}\right), \ldots, s_{n}\left(z_{n}\right)\right]^{T}$ with $s_{i} \in C^{1}[\mathbf{R},(-1,1)]$ where $s_{i}$ is the neuron activations and monotonically increasing for each $i=1,2, \ldots, n$.

The asymptotic stability of the zero solution of delaydifference system with multiple delays of Hopfield neural networks has been developed during the past several years. Much less is known regarding the asymptotic stability of the zero solution of the control discrete-time system of neural networks. Therefore, the purpose of this paper is to establish sufficient condition for the asymptotic stability of the zero solution of (1) in terms of certain matrix inequalities.

\section{PRELIMINARIES}

The following notations will be used throughout the paper. $\mathbf{R}^{+}$denotes the set of all non-negative real numbers; $\mathbf{Z}^{+}$ denotes the set of all non-negative integers: $\mathbf{R}^{n}$ denotes the 
Proc. of the Intl. Conf. on Advances in Applied science and Environmental Technology - ASET 2015.

Copyright (C) Institute of Research Engineers and Doctors, USA .All rights reserved.

ISBN: 978-1-63248-040-8 doi: 10.15224/ 978-1-63248-040-8-36

n-finite-dimensional Euclidean space with the Euclidean norm $\|$.$\| and the scalar product between x$ and $y$ is defined by $x^{T} y ; \quad \mathbf{R}^{n \times m}$ denotes the set of all $(n \times m)$-matrices; and $A^{T}$ denotes the transpose of the matrix $A$; Matrix $Q \in \mathbf{R}^{n \times n}$ is positive semidefinite $(Q \geq 0)$ if $x^{T} Q x \geq 0$, for all $x \in \mathbf{R}^{n}$. If $x^{T} Q x>0\left(x^{T} Q x<0\right.$, resp.) for any $x \neq 0$, then $Q$ is positive (negative, resp.) definite and denoted by $Q>0,(Q<0$, resp.). It is easy to verify that $Q>0,(Q<0, \quad$ resp. $) \quad$ iff $\quad \exists \beta>0$ : $x^{T} Q x \geq \beta\|x\|^{2}, \forall x \in \mathbf{R}^{n}$,

( $\exists \beta>0: x^{T} Q x \leq-\beta\|x\|^{2}, \forall x \in \mathbf{R}^{n}$, resp.) .

Lemma 1 The zero solution of difference system is asymptotic stability if there exists a positive definite function $V(x): \mathbf{R}^{n} \rightarrow \mathbf{R}^{+}$such that

$\exists \beta>0: \Delta V(x(k))=V(x(k+1))-V(x(k)) \leq-\beta\|x(k)\|^{2}$,

along the solution of the system. In case the above condition holds for all $x(k) \in V_{\delta}$, we say that the zero solution is locally asymptotically stable.

Lemma 2 [15] For any constant symmetric matrix $M \in \mathbf{R}^{n \times n}, \quad M=M^{T}>0$, scalar $s \in \mathbf{Z}^{+} /\{0\}$, vector function $W:[0, s] \rightarrow \mathbf{R}^{n}$, we have

$$
s \sum_{i=0}^{s-1}\left(w^{T}(i) M w(i)\right) \geq\left(\sum_{i=0}^{s-1} w(i)\right)^{T} M\left(\sum_{i=0}^{s-1} w(i)\right)
$$

We present the following technical fact and lemmas, which will be used in the proof of our main result.

\section{MAIN RESULTS}

In this section, we consider the sufficient condition for asymptotic stability of the zero solution $u^{*}$ of (1) in terms of certain matrix inequalities. Without loss of generality, we can assume that $u^{*}=0, S(0)=0$ and $f=0$ (for otherwise, we let $x=u-u^{*}$ and define $\left.S(x)=S\left(x+u^{*}\right)-S\left(u^{*}\right)\right)$.

The new form of (1) is now given by

$$
u(k+1)=-C_{i} u(k)+\sum_{i=1}^{m} B_{i} S\left(u\left(k-h_{i}\right)\right)
$$

Throughout this paper we assume the neuron activations $s_{i}\left(x_{i}\right), \quad i=1,2, \ldots, n$ is bounded and monotonically nondecreasing on $\mathbf{R}$, and $s_{i}\left(x_{i}\right)$ is Lipschitz continuous, that is, there exist constant $l_{i}>0, i=1,2, \ldots, n$ such that

$$
\left|s_{i}\left(v_{1}\right)-s_{i}\left(v_{2}\right)\right| \leq l_{i}\left|v_{1}-v_{2}\right|, \quad \forall v_{1}, v_{2} \in \mathbf{R} \text {. }
$$

By condition (3), $s_{i}\left(x_{i}\right)$ satisfy

$$
\left|s_{i}\left(x_{i}\right)\right| \leq l_{i}\left|x_{i}\right|, \quad i=1,2, \ldots, n
$$

Theorem 1 The zero solution of the delay-difference system (2) is asymptotically stable if there exist the symmetric positive definite matrices $P_{i}(k), G_{i}(k), W_{i}(k)$, and $L_{i}=\operatorname{diag}\left[l_{1 i}, \ldots, l_{n i}\right]>0, \quad i=1,2, \ldots, m$ satisfying the following matrix inequalities:

$$
\psi_{i}=\left[\begin{array}{cccccccccc}
(0,0) & 0 & 0 & \cdots & 0 & 0 & 0 & 0 & \cdots & 0 \\
0 & (1,1) & (1,2) & \cdots & \left(1, m_{i}\right) & 0 & 0 & 0 & \cdots & 0 \\
0 & (2,1) & (2,2) & \cdots & \left(2, m_{i}\right) & 0 & 0 & 0 & \cdots & 0 \\
\vdots & \vdots & \vdots & \ddots & \vdots & \vdots & \vdots & \vdots & \vdots & \vdots \\
0 & \left(m_{i}, 1\right) & \left(m_{i}, 2\right) & \cdots & \left(m_{i}, m_{i}\right) & 0 & 0 & 0 & \cdots & 0 \\
0 & 0 & 0 & \cdots & 0 & \left(m_{i}+1, m_{i}+1\right) & 0 & 0 & \cdots & 0 \\
0 & 0 & 0 & 0 & 0 & 0 & \left(m_{i}+2, m_{i}+2\right) & 0 & \cdots & 0 \\
\vdots & \vdots & \vdots & \vdots & \vdots & \vdots & 0 & \ddots & \ddots & \vdots \\
0 & 0 & 0 & 0 & 0 & 0 & \vdots & \ddots & \ddots & 0 \\
0 & 0 & 0 & 0 & 0 & 0 & 0 & \cdots & 0 & \left(2 m_{i}, 2 m_{i}\right)
\end{array}\right]<0,
$$

where

$$
\begin{aligned}
& (0,0)=C_{i}{ }^{T} P_{i}(k) C_{i}-P_{i}(k)+\sum_{i=1}^{m}\left(h_{i} G_{i}(k)+W_{i}(k)\right), \\
& (1,1)=L_{i} B_{1 i}{ }^{T} P_{i}(k) B_{1 i} L_{i}+\varepsilon_{1 i}{ }^{-1} L_{i} L_{i}-W_{1 i}(k), \\
& (1,2)=L_{i} B_{1 i}{ }^{T} P_{i}(k) B_{2 i} L_{i}+\varepsilon_{1 i}{ }^{-1} L_{i} L_{i}, \\
& (1, m)=L_{i} B_{1 i}{ }^{T} P_{i}(k) B_{m i} L_{i}+\varepsilon_{1 i}{ }^{-1} L_{i} L_{i}, \\
& (2,1)=L_{i} B_{2 i}{ }^{T} P_{i}(k) B_{1 i} L_{i}+\varepsilon_{1 i}{ }^{-1} L_{i} L_{i}, \\
& (2,2)=L_{i} B_{2 i}{ }^{T} P_{i}(k) B_{2 i} L_{i}+\varepsilon_{1 i}{ }^{-1} L_{i} L_{i}-W_{2 i}(k), \\
& (2, m)=L_{i} B_{2 i}{ }^{T} P_{i}(k) B_{m i} L_{i}+\varepsilon_{1 i}{ }^{-1} L_{i} L_{i}, \\
& (m, 1)=L_{i} B_{m i}{ }^{T} P_{i}(k) B_{1 i} L_{i}+\varepsilon_{1 i}{ }^{-1} L_{i} L_{i}, \\
& (m, 2)=L_{i} B_{m i}{ }^{T} P_{i}(k) B_{2 i} L_{i}+\varepsilon_{1 i}{ }^{-1} L_{i} L_{i} \\
& \left(m_{i}, m_{i}\right)=L_{i} B_{m i}{ }^{T} P_{i}(k) B_{m i} L_{i}+\varepsilon_{1 i}{ }^{-1} L_{i} L_{i}-W_{m i}(k), \\
& \left(m_{i}+1, m_{i}+1\right)=-h_{1 i} G_{1 i}(k),
\end{aligned}
$$


Proc. of the Intl. Conf. on Advances in Applied science and Environmental Technology - ASET 2015.

Copyright (C) Institute of Research Engineers and Doctors, USA .All rights reserved.

ISBN: 978-1-63248-040-8 doi: 10.15224/ 978-1-63248-040-8-36

$\left(m_{i}+2, m_{i}+2\right)=-h_{2 i} G_{2 i}(k)$,

$\left(2 m_{i}, 2 m_{i}\right)=-h_{m i} G_{m i}(k)$.

Proof Consider the Lyapunov function

$V(y(k))=V_{1}(y(k))+V_{2}(y(k))+V_{3}(y(k))$, where

$V_{1}(y(k))=x^{T}(k) P_{i}(k) x(k)$,

$V_{2}(y(k))=\sum_{i=1}^{m} \sum_{j=k-h_{i}+1}^{k}(h-k+i) x^{T}(j) G_{i}(k) x(j)$,

$V_{3}(y(k))=\sum_{i=1}^{m} \sum_{j=k-h_{i}+1}^{k} x^{T}(j) W_{i}(k) x(j)$

$P_{i}(k), G_{i}(k), W_{i}(k), i=1,2, \ldots, m$ being symmetric positive definite solutions of (5) and

$y(k)=\left[x(k), x\left(k-h_{1}\right), \ldots, x\left(k-h_{m}\right)\right]$.

Then difference of $V(y(k))$ along trajectory of solution of (2) is given by

$$
\Delta V(y(k))=\Delta V_{1}(y(k))+\Delta V_{2}(y(k))+\Delta V_{3}(y(k)) .
$$

From the above inequality it follows that:

$$
\begin{aligned}
& \Delta V \leq x^{T}(k)\left[C_{i} P_{i}(k) C_{i}-P_{i}+\sum_{i=1}^{m} h_{i} G_{i}(k)+W_{i}(k)\right. \\
& \left.+\varepsilon_{1 i} \sum_{i=1}^{m} \sum_{j=1}^{m} C_{i} P_{i}(k) B_{i} B_{j}^{T} P_{i}(k) C_{i}+\varepsilon_{i}^{-1} L_{i} L_{i}\right] x(k) \\
& +\sum_{i=1}^{m} \sum_{j=1}^{m} x^{T}\left(k-h_{i}\right)\left[L B_{i}^{T} P(k) B_{j} L+\varepsilon_{1}^{-1} L L\right] x\left(k-h_{j}\right) \\
& -\sum_{i=1}^{m} x^{T}\left(k-h_{i}\right) W_{i}(k) x\left(k-h_{i}\right) \\
& -\sum_{i=1}^{m}\left(\frac{1}{h_{i}} \sum_{j=k-h_{i}+1}^{k} x(j)\right)^{T}\left(h_{i} G_{i}(k)\right)\left(\frac{1}{h_{i}} \sum_{j=k-h_{i}+1}^{k} x(j)\right) \\
& =y^{T}(k) \psi_{i} y(k),
\end{aligned}
$$

By the condition (5), $\Delta V$ is negative definite, namely there is a number $\beta>0$ such that $\Delta V(y(k)) \leq-\beta\|y(k)\|^{2}$, and hence, the asymptotic stability of the system immediately follows from Lemma 1. This completes the proof.
Remark 1 Theorem 1 gives a sufficient condition for the asymptotic stability of delay-difference system (2) via matrix inequalities. These conditions are described in terms of certain diagonal matrix inequalities, which can be realized by using the linear matrix inequality algorithm proposed in [4]. But $\mathrm{Hu}$ and Wang [9] these conditions are described in terms of certain symmetric matrix inequalities, which can be realized by using the Schur complement lemma and linear matrix inequality algorithm proposed in [4].

\section{NUMERICAL SIMULATIONS}

Example 1 Let us consider the Hopfield-type neural networks with time varying delays (2) of the form

$$
u(k+1)=-C_{i} u(k)+\sum_{i=1}^{m} B_{i} S\left(u\left(k-h_{i}\right)\right),
$$

where the matrices are

$$
\begin{aligned}
\mathrm{C}_{i} & =\left(\begin{array}{ll}
1.9 & 1 \\
0.3 & 2
\end{array}\right), B_{i}=\left(\begin{array}{cc}
-0.9 & 0.3 \\
0.5 & -0.8
\end{array}\right), \varepsilon=0.5, \text { and } \\
h_{i} & =59 .
\end{aligned}
$$

Using the LMI Toolbox in MATLAB, we found that the LMIs in Theorem 1 are feasible and

$$
\begin{aligned}
P_{i} & =\left(\begin{array}{ll}
0.8973 & 0.2315 \\
0.2379 & 1.2376
\end{array}\right), G_{i}=\left(\begin{array}{ll}
1.3873 & 0.5697 \\
0.3279 & 0.6971
\end{array}\right), \\
W_{i} & =\left(\begin{array}{ll}
0.7325 & 0.1237 \\
0.3214 & 0.9668
\end{array}\right)
\end{aligned}
$$

are the set of solutions to the $\operatorname{LMIs}(5)$.

Therefore, the system is asymptotically stable. 
Proc. of the Intl. Conf. on Advances in Applied science and Environmental Technology - ASET 2015.

Copyright (C) Institute of Research Engineers and Doctors, USA .All rights reserved.

ISBN: 978-1-63248-040-8 doi: 10.15224/ 978-1-63248-040-8-36

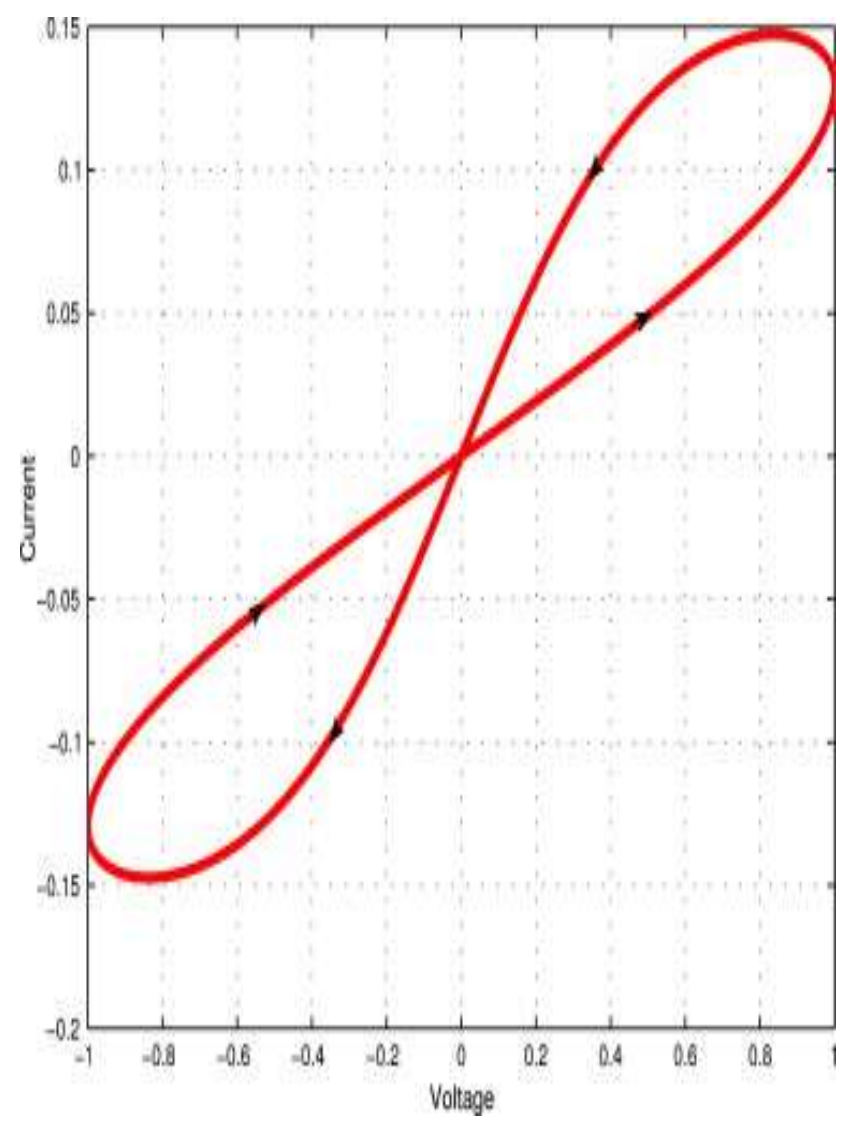

Fig. 1 Numerical simulation of a solution for the example 1.

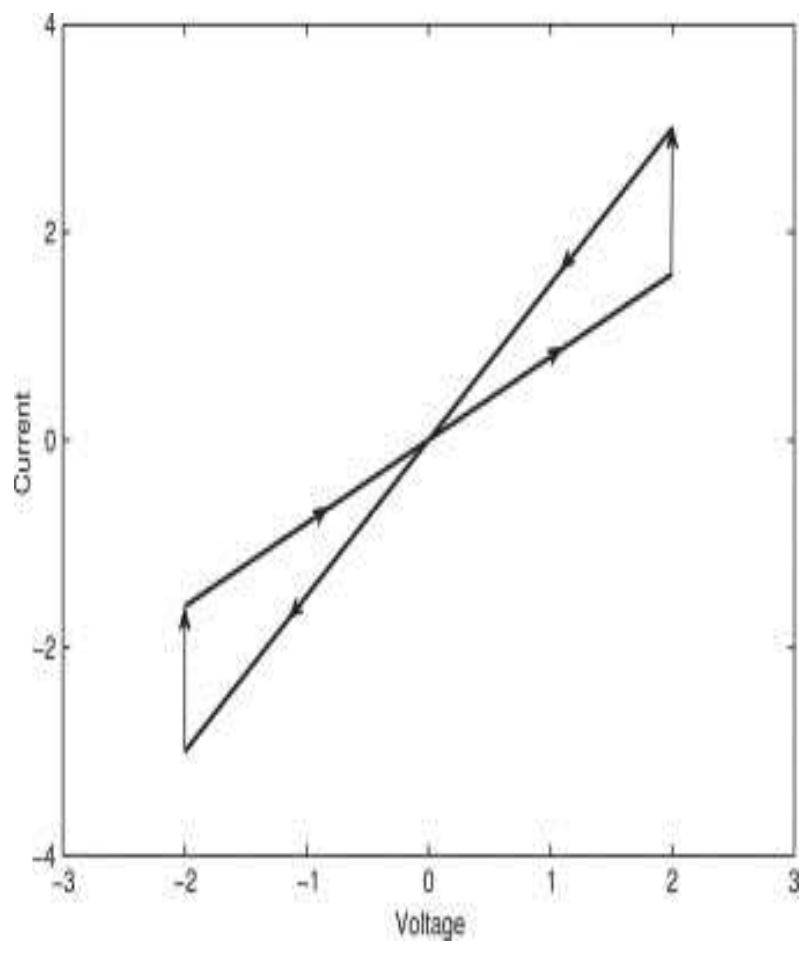

Fig. 2 Numerical simulation of a solution for the example 1.

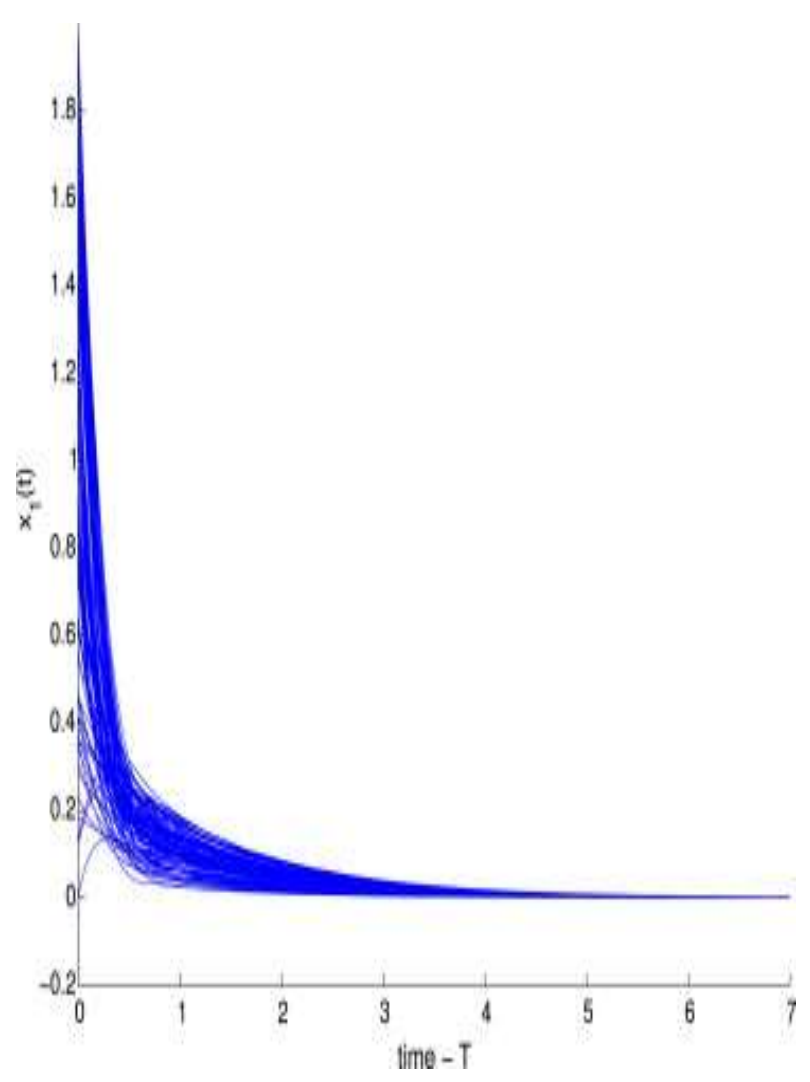

Fig. 3 Numerical simulation of a solution for the example 1.

For a given initial condition $x(\theta)=[1,1]^{T}$, convergence behavior of is shown in Fig. 1. As we can see from this figure, the steady state of nonlinear time varying delay-difference system is indeed asymptotically stable.

\section{V.CONCLUSION}

This paper was dedicated to the delay-dependent stability of delay-difference system with multiple delays of Hopfield neural networks. A less conservative LMI-based globally stability criterion is obtained with quadratic Lyapunov functional approach and free-weighting matrix approach for periodic delay-difference system with multiple delays of Hopfield neural networks.

\section{ACKNOWLEDGMENT}

This work was supported by the Office of Agricultural Research and Extension Maejo University Chiangmai Thailand and the Higher Education Commission and Faculty of Science, Maejo University, Thailand.

\section{REFERENCES}

[1] R.A. Chayjan and Y. Moazez, Estimation of paddy equilibrium moisture sorption using ANNs. J. Applied Sci., Vol. 8 (2008), pp. 346-351. 
[2] V. N. Phat and K. Ratchagit, Stability and stabilization of switched linear discrete-time systems with interval time-varying delay, Nonlinear Analysis: Hybrid Systems, Vol. 5 (2011), pp. 605-612.

[3] K. Ratchagit, Asymptotic stability of delay-difference system of Hopfield neural networks via matrix inequalities and application, International Journal of Neural Systems, Vol. 17 (2007), pp. 425-430.

[3] V. N. Phat, Y. Khongtham and K. Ratchagit, LMI approach to exponential stability of linear systems with interval time-varying delays, Linear Algebra and its Applications, Vol. 436 (2012), pp. 243-251.

[4] M. Rajchakit and G. Rajchakit, Mean square exponential stability of stochastic switched system with interval time-varying delays, Abstract and Applied Analysis, Volume 2012 (2012), Article ID 623014, 12 pages. http://dx.doi.org/10.1155/2012/623014

[5] J. Li, Y. F. Diao, M. D. Li and X. Yin, Stability analysis of Discrete Hopfield Neural Networks with the nonnegative definite monotone increasing weight function matrix, Discrete Dynamics in Nature and Society, Vol. 2009 (2009), Article ID 673548, 10 pages.

[6] J. Li, W. G. Wu, J. M. Yuan, Q. R. Tan and X. Yin, Delay-dependent stability criterion of arbitrary switched linear systems with time-varying delay, Discrete Dynamics in Nature and Society, Vol. 2010 (2010), Article ID 347129, 16 pages.

[7] J. L. Liang, Z. D. Wang, Y.R. Liu and X. H. Liu, Robust Synchronization of an Array of Coupled Stochastic Discrete-Time Delayed Neural Networks, IEEE Trans. on Neural networks, Vol. 19 (2008), pp.1910-1921.

[8] Y. R. Liu, Z. D. Wang and X. H. Liu, Asymptotic stability for neural networks with mixed time-delays: the discrete-time case, Neural Networks, Vol. 22 (2009), pp. 67-74.

[9] M. Rajchakit, P. Niamsup and G. Rajchakit, A switching rule for exponential stability of switched recurrent neural networks with interval timevarying delay, Advances in Difference Equations, Vol. 2013 (2013:44).

[10] C. Y. Lu, H. H. Tsai, T. J. Su, J. Tsai and C. W. Liao, A delay-dependent approach to passivity analysis for uncertain neural networks with timevarying delay, Neural Process Lett, Vol. 27 (2008), pp. 237-246.

[11] K. S. Narendra and J. Balakrishnan, Adaptive control using multiple models, IEEE Transactions on Automatic Control, vol. 42, no. 2, pp. 171$187,1997$.

[12] K. Ratchagit, Asymptotic stability of nonlinear delay-difference system via matrix inequalities and application, International Journal of Computational Methods, vol. 6 (2009), pp. 389-397.

[13] Y. Fu and T. Chai, Nonlinear multivariable adaptive control using multiple models and neural networks, Automatica, vol.43, no. 6, pp. 11011110, 2007.

[14] M. Rajchakit and G. Rajchakit, LMI approach to robust stability and stabilization of nonlinear uncertain discrete-time systems with convex polytopic uncertainties, Advances in Difference Equations, vol. 2012 (2012:106).

[15] G. C. Goodwin and K. S. Sin, Adaptive Filtering Prediction and Control, Prentice-Hall, Englewood Cliffs, NJ, USA, 1984.

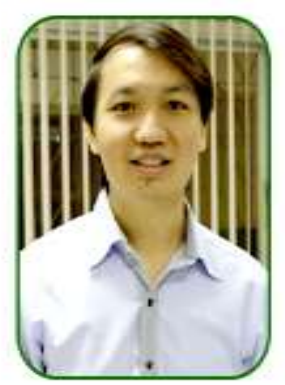

Grienggrai Rajchakit received his MSc degrees in applied mathematics at the Chiang Mai University, Thailand in 2001 and PhD degrees in applied mathematics at the King Mongkut's University, Thailand in 2007, respectively. He is the author/co-author of 89 refereed journal papers. In 2007 he joined the Department of Mathematics, Maejo University Thailand as a Lecturer, where he became an Assistant Professor in 2013. His research areas include mathematical analysis, stability analysis, difference equations, neural networks. 